\title{
The Design and Implementation of the Remote
}

\section{Centralized-Monitoring System of Well-Control Equipment Based on RFID Technique}

\author{
Bin Luo ${ }^{1}$,Jiarui Liu ${ }^{2}$, Junlan $\mathrm{Li}^{3}$ and haibo Liang ${ }^{1}$ \\ ${ }^{1}$ School of Mechatronic Engineering, Southwest Petroleum University, Chengdu 610500,China \\ ${ }^{2}$ Sichuan BOMCO Special Vehicle Manufacturing Co. Ltd, Guanghan 618300, China \\ ${ }^{3}$ Southwest Branch, Engineering Design Co., CNPC, Chengdu 610413, China
}

\begin{abstract}
At present, in domestic for the management of well control equipment continue to the traditional way of nameplates identifies and paper-based registration, there are many issues like the separation of data information of device, easy lose, difficult query, confused management and many other problems, which will make the problem device into the well field, and then resulting in well control runaway drilling accident. To solve the above problems, this paper put forward to the integrated remote centralized-monitoring management mode of the well-control equipment. Taking the advantages of IOT technology, adopting the RFID technology, and combining with the remote transmission, this paper designs the remote centralized-monitoring system of well-control equipment based on RFID, which realizes the intelligent management of well-control equipment and meets the actual demand of the well-control equipment safe use and timely scheduling, and it has the ability of field application.
\end{abstract}

Keywords. Internet of Things; RFID, Well-control Equipment, Remote transmission, Remote Centralized-monitoring Management.

\section{Introduction}

Well control equipment is a safety measure of pressure control and safety drilling for oil and gas Wells. The well control equipment that is high quality, reliable, and meet the requirements of the target well, Not only can effectively implement well control, reduce drilling accidents, exploitation of oil and gas reached safety requirements, but also can ensure drilling quality and reduce non-work time.

However, since domestic well site geographically isolated and wide distributed, the use of well control equipment traditional nameplate, paper-based registration results in well control equipment trans-regional, intersect oral distribution, equipment and data separation, easy to lose and difficult inquiry. Even leads to well control equipment scheduling is difficult, and problem equipments easily flow into the well site, So that once the overflow, well kick occurs, you can not rely on well control equipment to achieve the purpose of preventing blowout, eventually leading to a vicious drilling accidents [1]. At present, as network technology has improved, network in the materials management, logistics, manufacturing and other fields has been widely used [2]. In other countries, a growing number of oil companies use network technology to achieve the construction of digital oilfield, intelligent field [3] - [6], and deal with the problems in 
the time of oilfield material using, perform modernization, such as centralized control and management of oil supplies. While the domestic oil industry, the researches and applications of network, mainly concentrate in the construction of wireless sensors and digital oil fields [7] - [9], management of oil supplies such as well control equipment, still remain in the traditional plate identification, paper registration management mode, unable to resolve problem of the well control equipment effective recognition and decentralized distribution fundamentally.

In this paper, well control equipment integrated remote centralized monitoring and management, the use of Networking technology, the use of RFID technology, combined with remote data transmission, Design and develop a well control equipment remote centralized control management system based on the network RFID. The system meets the actual demand of the safe use of well control equipment, real-time scheduling, changes the use of the traditional well control equipment nameplate, paper registration, and achieve that problem equipments can not flow into the well site, materials and equipment scheduling timely and well control equipment centralized monitoring and intelligent management. It is of great significance for drilling site providing reliable, reasonably well control equipments to ensure safe and high quality drilling, reduce the risk of accidents and increase economic efficiency.

\section{Integrated remote centralized monitoring and well control equipment management}

With the construction of the digital oil field, the networking technology in the oil industry has been widely used in development, but well control equipment still using the traditional nameplate, paper-based registration usage modes. Network could not be used for well control equipment remotely centralized control management to form a mature and perfect technology system, can not meet the actual demand on safe use of well control equipment and timely dispatch.

For the status of the management of well control equipment in oil industry, analyze the feasibility of networking well control equipment remote centralized control management from three aspects of the perception layer, transport layer, application layer, and the specific performance in the following areas:

(1) Use networking perception layer enables accurate identification of well control equipment, to avoid problems equipment into the well field.

(2) Use networking transport layer can provide effective interactive data transmission channels, to achieve timely and well control equipment scheduling.

(3) Use networking application layer enables centralized monitoring and well control equipment, digital and intelligent management.

Feasibility Analysis realized by the use of networking technology well control equipment remote centralized control management can be seen, through using RFID technology, combined with long-distance transmission can be realized from the well control equipment inspection and maintenance, inventory to a closed-loop drilling site centralized monitoring and management mode. In this integrated remote centralized monitoring and management mode, each well control equipment could be dated its source and ensure the reliability. Accordingly, to solve well control equipment identification, decentralized distribution, the device status is difficult to determine and so on, to prevent the problem device into the well site, timely dispatch equipment, centralized monitoring, remote centralized control management integrated intelligent management purposes, to provide protection for safe and efficient drilling.

\section{The overall system design}

According to well control equipment integrated remote centralized monitoring and management model, the well control equipment remote centralized control management system based on network consists of the perception layer (data acquisition system), the transport layer (Remote Data Transmission System) and application layer (well control equipment centralized control management system) constitute the overall design of the system shown in Figure 1. 


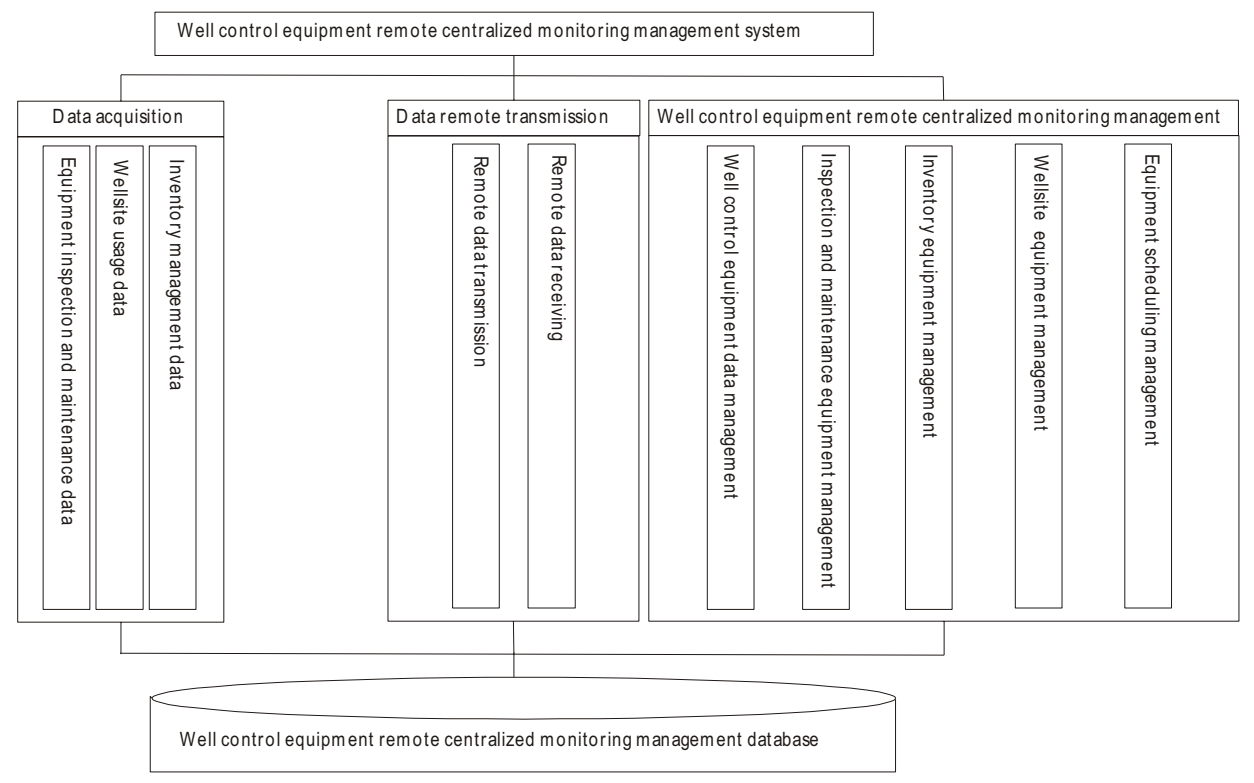

Fig.1 Overall design scheme

\section{System features modular design}

\subsection{Perception layer}

The main role of remote sensing layer of well control equipment centralized control management system is unique identification, automatic identification of well control equipment. Perception by reading and writing RFID tag data layer, it is possible to achieve the well control equipment to effectively identify and track, to achieve the purpose of providing high-quality, reliable and well control equipment to the well site. For questions of perception layer for high frequency (HF) and ultra high frequency (UHF) RFID data collection, transmission, storage and so on, through the serial communication, Windows Sockets network programming interface to achieve sensing layer data collection and transmission. The design block diagram of sensing layer in well control equipment remote centralized control management system is shown in Figure 2 .

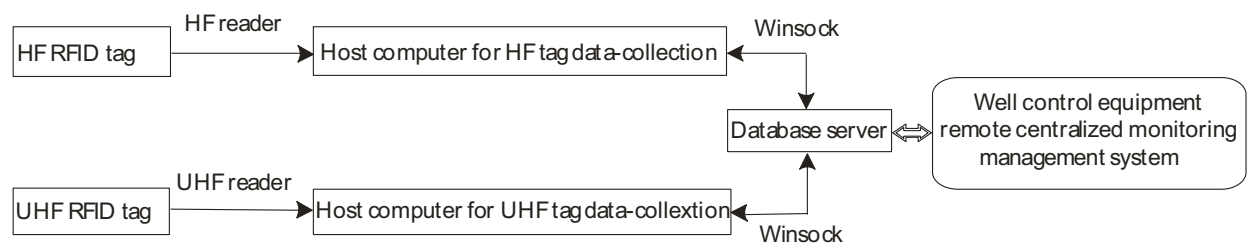

Fig.2 Block diagram of perception layer design

System perception layer use High Frequency (HF) and Ultra High Frequency (UHF) reader for the respective electronic tag to collect data or update storage information, Data collected or updated is sent to the management server to well control equipment by the host computer, and achieve the data and information of well control equipment management applications. Perception layer data acquisition and transmission program uses $\mathrm{C}$ \# language, under the Visual Studio 2010 integrated development environment, it enables the development of data acquisition software systems and achieve data collection, and two-way communication between PC and the server. 


\subsection{Transport layer}

The transport layer for well control equipment remote centralized control management system mainly communicates data between management center with well control equipments well site, In order to achieve the integration of centralized monitoring and management of well control equipment. According to the overall system design preferred remote communication, in use of CDMA / GPRS wireless communication network, design a suitable for well control equipment management center and well site to achieve interactive remote data transmission scheme, Transport Layer system design block diagram is shown in Figure 3.

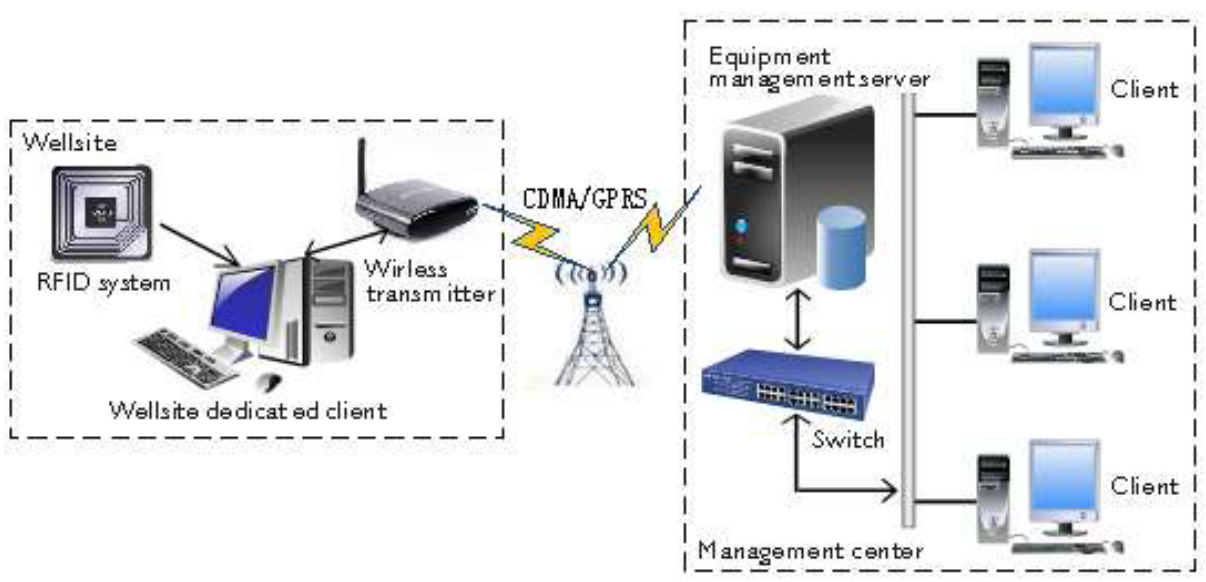

Fig.3 Block diagram of transport layer design

\subsection{Application layer}

Design of well control equipment remote centralized control management system application layer is based on a system of perception layer and transport layer. Application layer software system can combine data collected by layers sensing organic, to prevent the problem device flow into the well field, well control equipment are dispatched in time, integration of centralized monitoring, intelligent management. Application layer software system faces well control equipment management, between the various software functional subsystems, both independent, and can combine into one, to achieve the orderly management of well control equipment.

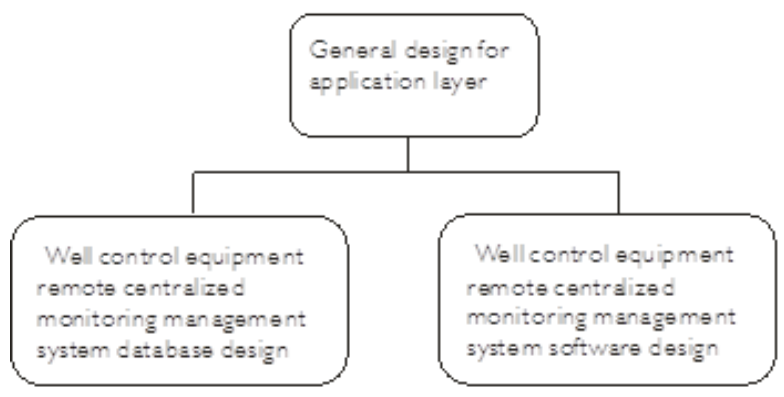

Fig.4 Block diagram of application layer design

\section{The design of well control equipment remote centralized control management system software}

Well control equipment remote centralized control management software is developed based on SQL Server 2008 database management platform and $\mathrm{C}$ \# high-level programming language, mainly to complete, well control equipment 
basic information management, equipment inventory management, inspection and maintenance management, well site equipment management, system management, and other functions.

\subsection{Basic information management subsystem}

Well control equipment remote centralized control management software, basic information management subsystem, is used for setting well control equipment, well site, alarm reminders and other public categories data, in order to prevent the operator mistakenly fill. The subsystem consists of well control equipment basic data settings, setting the alarm to remind and other data settings of three functional modules, usually, it is operated by an administrator.

\subsection{Inspection and maintenance management subsystem}

Well control equipment inspection and maintenance management subsystem is used for checking for well control equipment, repairing and maintaining management. Well control equipment inspection management mainly finish safety inspection records and inquiries for well control equipment, housing and others; equipment maintenance management is used to restore faulty equipment functions management; well control equipment maintenance is maintaining equipments daily, such as lubrication, replacement of aging components, periodic inspections to ensure well control equipment status, and extend equipment life.

\subsection{Equipment inventory management subsystem}

Device inventory management subsystem is used to implement well control equipment in the library and out of storage management. Device inventory management files operate and manage all of inventory and equipment information, and it is able to view all equipment in the library, and print the related reports; in and out of storage management of device can operate well control equipment in and out of storage, Ordered to complete well control equipment inventory management, to ensure equipment inventory data is correct.

\subsection{Well site equipment management subsystem}

Well site equipment management subsystem has functions of well site equipment records management, query the device information, well site equipment statistical. Meanwhile, the subsystem has a well site equipment inspection management, the equipments transported to well site can be completed the inspection work as required, Avoid the problem equipments further be flew into the well field to be used; well site equipment management logs are in charge of the well field equipment inspection, everyday use, maintenance management by selecting notes category; well site equipment scheduling management is used to achieve the target device that wellsite remote desired, recover well site construction in time.

\section{Conclusion}

This article uses the advantage of network technology, and RFID technology, combined with remote data transmission, and puts forward to the integrated remote centralized-monitoring management mode of the well-control equipment. Design and develop a set of well control equipment remote centralized control management system collected of well control equipment data acquisition, remote transmission, centralized monitoring as one. After laboratory tests, the running result of system is stable, reliable, has a ability to field applications. Development and application of the system will change management of the traditional well control equipment nameplate paper-based registration, effectively prevent the problem device into the well field, and well control equipments meet the actual needs of safe use or real-time scheduling, and it can provide strong technical support for well control equipment digital intelligent management, security of well site drilling, reducing the risk of well control accidents, improving economic efficiency. 


\section{References}

1. Wu J J. Monitoring and pre-warning method study on drilling engineering accidents. Mud Logging Engineering, 2006,3(1):53-55,70

2. Jiang Y B, You D Z, Ge Y J. Study on Popularization and Application of IOT in the Logistics Industry, Logistics Sci-Tech, 2012(10): 101-103.

3. van der Steen E, Knoppe R. BSP: An evolution from Smart Wells to Smart Fields. SPE100710, 2006.

4. L Ge, Pan Hu, Xiaohui Xie, Ze Hu, Qiang Zeng, Junbi Liao. Study on High Precision Swill-cooked Dirty Oil Detection System Based on Date Fusion Technology,Oxidation Communications,2016, book 1, 39,317-330

5. Shepard J S, Perkin G S, Denny LA. Electronic Identification of Drillstem and Other Components Used in Harsh Environments Proves Successful.SPE/IADC25774,Drilling Conference. Society of Petroleum Engineers, 1993.

6. L Ge, Ze Hu, Chen Ping et al. Research on Overflow Monitoring Mechanism Based on Downhole Microflow Detection, Mathematical Problems in Engineering, Volume 2014:1-6.

7. L Ge, Zhengyin Wang, Kui Deng, Qiang Zeng, Xi Wang, Xiaoshuang Chen, Junbi LIAO. Research on the Oil Life Estimation and Detection Method, Journal of the Balkan Tribological Association, 2015, book 4,21, 897-911.

8. L Ge, Qiang Zeng, Zhengyin Wang, Xiaohui Xie,Junbi Liao, Junlan Li. Research on Moisture Content Measuring Device of Edible Oil Based on Capacitance Detection Mechanism,Oxidation Communications,2016, book 1, $39,240-248$

9. Wei Ming, Lu Yongjie WangHaiguo,et al.Practice of Internet of Things in Oilfield Enterprise. Journal of Chongqing University of Science and Technology(Social Sciences Edition),2013, 15(2): 154-158. 